\title{
Editorial
}

Wissenschaftliche Evaluation befindet sich immer im Spannungsfeld zwischen den Anforderungen, die aus der Wissenschaft an sie gestellt werden - dies betrifft insbesondere die Methodik des Vorgehens respektive die Genauigkeitsstandards - und den Bedingungen, die sich im Feld stellen, respektive den Logiken, denen das Feld unterliegt. Hier eine Balance zu finden, die beiden Seiten Rechnung trägt, d.h. den wissenschaftlichen Anforderungen zu entsprechen und gleichzeitig die Praxisbedingungen ausreichend zu berücksichtigen, ist eine der größten Herausforderungen für Evaluator(inn)en. Dies zeigt sich bei allen vier Originalbeiträgen des vorliegenden Hefts der Zeitschrift für Evaluation, obwohl diese sich inhaltlich mit sehr unterschiedlichen Themen und Fragestellungen beschäftigen. In allen vier Beiträgen werden diese Herausforderungen auch explizit thematisiert und aufgezeigt, wie man die notwendige Balance herstellen kann.

Der erste Beitrag von Yasmin Balibasa und Christian Diller widmet sich der wirkungsorientierten vergleichenden Evaluation. Aufbauend auf theoretischen Überlegungen dazu wird die vergleichende Evaluation der Wirkungen von drei lokalen Masterplänen Industrie vorgestellt. Innerhalb eines „Evaluability-Assessments“ wurden von den Autor(inn)en im ersten Schritt, als Voraussetzung für den Vergleich, die implizit vorhandenen Wirkungsannahmen in einem einfachen logischen Grundmodell herausgearbeitet. Die durchgeführte Systematisierung der Masterpläne zeigte, dass diese unterschiedliche Entwicklungen bedingen und anstoßen. Der Erfolg eines solchen Plans wird jedoch von unvorhersehbaren Ereignissen und externen Faktoren beeinflusst (z.B. Wirtschaftskrisen oder Klimawandel), die gemäß den Autor(inn)en weder messbar noch prognostizierbar sind. Als Problem mit Blick auf die oben angesprochene Balance werden von den Autor(inn)en die Argumentationen der Politik, die auf einfachen Wirkungsannahmen basieren, genannt. Ihrer Ansicht nach muss sich die Wissenschaft jedoch dieser politischen Logik stellen, die Indikatoren der Politik aufgreifen und auf deren beschränkte Aussagekraft hinweisen. Als informelles Instrument unterliegt ein Masterplan auch keinen rechtlichen Rahmenbedingungen und es besteht keine Verbindlichkeit zur Maßnahmenumsetzung. Dennoch setzen solche Masterpläne nach Meinung der beiden Autor(inn)en Rahmenbedingungen für industriepolitische Maßnahmen und ergänzen Bereiche der Stadtentwicklung. Sie empfehlen daher weiterführende Forschungen, die die unterschiedlichen Interessen von Akteur(inn)en bei der Erarbeitung der Pläne sowie die Aushandlungs- und 
Konsensbildungsprozesse tiefergehend analysieren und damit die Lücke zwischen Evaluations- und Policy-Forschung weiter schließen.

Im zweiten Beitrag von Kira Hower, Holger Pfaff, Jörg Kons, Sandra Groß und Lena Ansmann werden die Ergebnisse der Evaluation des FITKIDS-Programms zur Kinderorientierung in Drogenberatungsstellen vorgestellt. Das Organisationsund Personalentwicklungsprogramm FITKIDS hat zum Ziel, die gesunde Entwicklung von Kindern drogenabhängiger, suchtkranker Eltern zu stärken. Die in dem Beitrag vorgestellte Evaluationsstudie EvaFit untersucht die Implementierung von FITKIDS in 15 Sucht- und Drogenberatungsstellen hinsichtlich Strukturen, Prozessen, Kenntnissen und Kompetenzen. Die zentrale methodische Herausforderung, die sich durch die Rahmenbedingungen den Evaluator(inn)en in diesem Projekt stellte, war die freiwillige Teilnahme der Sucht- und Drogenberatungsstellen. Es gab keine Möglichkeit zur Randomisierung. Die Autor(inn)en begegneten diesem Problem dadurch, dass sie Pilotstandorte als Referenzgruppe heranzogen. Ein weiteres Problem mit Blick auf die Interpretation der Ergebnisse, das im Beitrag diskutiert wird, war die Erhebung der Daten mittels Selbstauskünften. Auf Basis der Befunde ziehen die Autor(inn)en die nachvollziehbare Schlussfolgerung, dass das ,Endziel' von FITKIDS, nämlich das gesunde Aufwachsen von Kindern, erst dann untersucht werden kann, wenn das Programm erfolgreich in den Beratungsstellen implementiert ist. Die Evaluation dieses Endziels soll in einer Folgestudie von EvaFit erfolgen.

Der dritte Originalbeitrag, der von Steffen Greve und Claus Krieger verfasst wurde, hat die nutzenfokussierte Evaluation eines inklusiven Angebots im Vereinsund Wettkampfsport zum Thema. Konkret geht es um die Evaluation der inklusiven Handballinitiative Freiwurf Hamburg (NEvaFrei) gemäß des Ansatzes der ,Utilization-Focused Evaluation' nach Patton. Die Autoren betonen den Vorteil einer hohen Flexibilität im Forschungsprozess für Evaluierende. Mit Blick auf die oben angesprochene Balance erfordert diese Flexibilität jedoch auch hohe Transfer- und Übersetzungsleistungen und führt zu einer Reduktion dessen, was Forschung und Evaluation eigentlich leisten könnten. Da sämtliche Entscheidungen über den Verlauf von NEvaFrei mit den Nutzer(inne)n getroffen wurden, konnten bzw. können nicht alle Ideen der Evaluierenden für den Forschungsprozess umgesetzt werden. Während die Evaluations(zwischen)ergebnisse innerhalb eines planbaren, vorgegebenen zeitlichen Rahmens vorlagen bzw. -liegen, ist die Verarbeitung dieser in der Praxis schwer zeitlich berechenbar. Die Autoren halten abschließend fest, dass, sofern man Nutzenfokussierung ernst nimmt, das Evaluationsprojekt kein konkretes Ende haben kann und daher auch für das Projekt NEvaFrei noch kein Schlusspunkt abzusehen ist. Insgesamt zeigt dieser Beitrag die Potenziale aber auch die Grenzen des forschungsmethodischen Vorgehens im Rahmen der nutzenfokussierten Evaluation im konkreten Setting auf.

Der vierte und letzte Beitrag stammt von Franziska Heinze, Stefanie Reiter, Ulrike Berg-Lupper, Katharina Wach und Stephanie Riedle. Er widmet sich den multiplen Handlungsanforderungen an Evaluierende bei der Evaluation komplexer (sozial-)politischer Programme. Konkret geht es um die unterschiedlichen Rollen von und Handlungsanforderungen an die Evaluierenden. Anhand von drei Beispielen aus der Evaluationspraxis werden von den Autorinnen Strategien der praktischen Bear- 
beitung multipler Handlungsanforderungen reflektiert. Anhand der drei Beispielprojekte, in denen sich für die Evaluierenden ähnliche Herausforderungen stellten, wird verdeutlicht, dass sich multiplen Handlungsanforderungen nicht durch eine frühzeitige Festlegung und konsequente Ausübung von spezifischen, feststehenden Rollen der Evaluierenden begegnen lässt. Vielmehr haben sich (um den Praxisbedingungen der komplexen Programme Rechnung zu tragen) gemäß den Autorinnen folgende drei Strategien als hilfreich herausgestellt: die Schaffung und Gestaltung geeigneter Aushandlungsorte, die Etablierung von Formaten zur (Auf-)Klärung von Erwartungen und Ansprüchen der Handlungspartner, und eine situativ angepasste, wandelbare und zum Teil (personell getrennte) gleichzeitige Übernahme von Rollen von Evaluierenden. Die Autorinnen empfehlen die entsprechenden Prozesse zu beschreiben und zu reflektieren sowie im Rahmen von Metaevaluationen aufzuarbeiten.

Der Information-\&-Service-Teil dieses Hefts enthält drei Beiträge, die sich ebenfalls sehr interessanten Themen widmen. Dawid Stawski und Magdalena Przybysz-Stawska beschäftigen sich im ersten Beitrag mit einem in der Wissenschaft intensiv und kontrovers diskutierten Thema: Wie die akademischen Leistungen von Hochschulpersonal in Europa vergleichend bewertet werden können. Nach einer Diskussion von häufig verwendeten qualitativen und quantitativen Bewertungen schlagen sie einen Algorithmus vor, der beide Aspekte berücksichtigt. Da dies ein Beitrag zur Rubrik Debatte ist, sind alle interessierten Leser(innen) der Zeitschrift für Evaluation aufgefordert, auf die gebotenen Argumente mit eigenen Beiträgen zu reagieren. Der Praxisbericht von Elisabeth Wachsmuth, Henrik Brinkmann und Jan Hense hat die Wirkungsorientierung in der Non-Profit-Programmarbeit zum Thema. Anhand eines Praxisbeispiels aus einer großen deutschen Non-Profit-Organisation wird die Umsetzung verschiedener Maßnahmen, wie Schulungen und der Einsatz von Wirkungsmodelltypen dargestellt, die zu mehr Wirkungsorientierung in der Programmarbeit beitragen können. Abschließend diskutieren die Autor(inn)en, inwiefern die Erfahrungen des Praxisbeispiels allgemein für Non-Profit-Organisationen relevant und übertragbar sein können. Der dritte Beitrag im Information-\&-ServiceTeil ist ein Praktikumsbericht. Er stammt von Anja Berhorst und widmet sich der Evaluationskultur in Costa Rica.

Das vorliegende Heft enthält auch eine von Wolfgang Beywl verfasste Rezension der zweiten Edition des von Marvin C. Alkin und Anne T. Vo verfassten Buchs „From A to Z“, das 2018 bei Guilford Press New York erschienen ist. Laut Wolfgang Beywl leistet Alkin einen originellen, mit seiner Evaluationsbiographie verbundenen Beitrag dazu, den komplexen, emergenten, stets Iteration und Neupositionierung erfordernden Prozess der Evaluationsplanung nachvollziehbar darzustellen. Als kleinere Schwächen werden das Fehlen von Literaturangaben und das Vorliegen einiger Inkonsistenzen angeführt. Laut Beywl ist das Buch jedoch weniger ein Lehrbuch für Beginner(innen) im Feld der Evaluation als ein Bildungsbuch für Fortgeschrittene.

Die Beiträge der DeGEval ...Info illustrieren die kontinuierliche und erfolgreiche Arbeit, die in den Arbeitskreisen respektive im Nachwuchsnetzwerk der DeGEval geleistet wird. Lisa Ringhofer berichtet über die dritte Session des AK Methoden in der Evaluation, die sich - diesmal im Rahmen der Jahrestagung 2018 in Dresden 
- einer politikfeldübergreifenden Diskussion zu methodischen Standards der Evaluation widmete. Ziel dieses Diskussionszyklus ist es, mehr über aktuelle methodische Trends, Herausforderungen, Gemeinsamkeiten und Unterschiede in den jeweiligen Arbeitskreisen der DeGEval herauszuarbeiten und mögliche Synergieeffekte und deren Innovationspotenzial auszuloten. Das Nachwuchsnetzwerk der DeGEval organisierte im März 2019 sein bereits achtes Forschungs- und Praxiskolloquium. Michael Bigos, Ina Dupret, Melanie Hörth, Sylvia G. Hundenborn und Katharina Papke berichten über dieses Kolloquium, das unter dem Thema „Evaluationspraxis fördern: Der Peer-to-Peer-Ansatz in der Nachwuchsarbeit" stand. Die wechselseitige Beratung ist deshalb ein zentrales Anliegen des Netzwerks, da durch den interdisziplinären Charakter von Evaluation oftmals keine ausgeprägten Unterstützungsstrukturen zu diesem Feld innerhalb der Fachbereiche, Institute oder Lehrstühle vorhanden sind. Der dritte Bericht stammt vom AK Entwicklungspolitik und Humanitäre Hilfe der DeGEval. Die Frühjahrstagung 2019 dieses Arbeitskreises widmete sich dem Thema Evaluation von Capacity Development. Laut den Autor(inn)en Stefanie Wein, Thorsten Bär, Saron Dawit, Susanne von Jan und Maximilian Schmid ist Capacity Building seit geraumer Zeit fester Bestandteil von zahlreichen Projekten und Programmen der Entwicklungspolitik; gleichzeitig fehlen jedoch eine allgemein akzeptierte Definition und entsprechende Messungen. Ziel der Frühjahrstagung war es daher, zu erörtern, mit welchen verschiedenen Methoden und Vorgehensweisen die Wirkungen von Capacity-Building-Maßnahmen auf unterschiedlichen Ebenen gemessen werden können unter Berücksichtigung des entwicklungspolitischen Kontexts. Angela Wroblewski berichtet im vierten Beitrag über die Frühjahrstagung 2019 des AK Gender Mainstreaming, die dem Thema „Genderkompetenz-Trainings für Evaluierende“" gewidmet war. Ziel dieser Tagung war es gemeinsam mit eingeladenen Expert(inn)en für Genderkompetenz-Trainings zu diskutieren, inwieweit bestehende Angebote auch für an Evaluationen Beteiligte geeignet sind bzw. welche spezifischen Adaptierungen notwendig sind. Schließlich widmet sich der fünfte Beitrag der DeGEval ...Info von Karoline Rodriguez, Jan Wessels, Marianne Kulicke und Sascha Ruhland dem diesjährigen Frühjahrstreffen des AK FTI, in dessen Rahmen anhand aktueller Projektbeispiele methodische Herausforderungen und neue Herangehensweisen bei der Evaluation diskutiert wurden.

Darüber hinaus enthält dieses Heft die Ausschreibung des Nachwuchspreises der DeGEval sowie die Ankündigung der nächsten Jahrestagung.

Im Namen der Herausgeber(innen) der Zeitschrift für Evaluation bedanke ich mich sehr herzlich bei den Autor(inn)en für ihre interessanten Beiträge und bei den Gutachter(inne)n für ihre Unterstützung im Qualitätssicherungsprozess. Den Leser(inne)n der Zeitschrift für Evaluation wünsche ich wie immer eine spannende Lektüre.

\section{Christiane Spiel}

Für die Herausgeberinnen und Herausgeber 